\title{
Antimicrobial-Resistant Evolution and Global Spread of Enterococcus faecium Clonal Complex (CC) 17: Progressive Change from Gut Colonization to Hospital-Adapted Pathogen
}

\author{
Zixin Peng'; Lin Yan'; Shuran Yang'; Dajin Yang ${ }^{1, *}$
}

\begin{abstract}
For a long time, Enterococcus faecium (E. faecium) was thought to be a commensal strain in human and animal digestive tracts. However, over the past three decades, some unique E. faecium clones rapidly acquired multiple antimicrobial resistance (AMR), which led these clones to survive hospital environments and become a hospital-adapted E. faecium clonal complex (CC) 17. Since the adaptation of these clones to changes in habitat, vancomycin-resistant $E$. faecium CC17 has emerged as the leading cause of hospitalacquired infections worldwide. This epidemic hospitaladapted lineage has diverged from other populations approximately 75 years ago. The CC17 lineage originated from animal strains, but not human commensal lines. We reviewed the evolutionary progress and the molecular mechanisms of E. faecium CC17 from a gut commensal to a multi-antimicrobial resistant nosocomial pathogen.
\end{abstract}

\section{INTRODUCTION}

Enterococcus faecium (E. faecium) is becoming one of the leading causes of hospital-acquired infections. However, in the past few decades, E. faecium was thought to be a commensal of the animal digestive tract, or even a probiotic, and an important cause of nosocomial infections. The hospital-adapted lineage of E. faecium clonal complex (CC) 17 has caused enormous burdens for hospitals, which can cause severe morbidity and mortality (1). The resistance to ampicillin, vancomycin, and other microbials has made this lineage difficult to treat in hospital settings.

E. faecium is mainly classified into two types: one is community/commensal-associated E. faecium CC94, whereas the other is hospital-adapted $E$. faecium $\mathrm{CC} 17$ (2). By average nucleotide identity analysis (ANI), E. faecium CC17 and CC94 have nucleotide differences totaling more than $5 \%$, and thus, above the threshold used for species identification (3). Compared with $E$. faecium CC94, the CC17 lineage contains pathogenicity islands (PAI) and plasmids or other mobile genetic elements (MGEs) associated with antimicrobial resistance (AMR), virulence, and/or colonization (4). This pathogenic lineage likely emerged from animal strains approximately 80 years ago, as a result of using antimicrobials to treat humans and animals. As such, an understanding of the progressive changes and the molecular mechanisms of this rapidly evolving lineage is of substantial interest and the first step in prevention.

\section{OCCURRENCE OF E. FAECIUM CC17}

The evolutionary trajectories of E. faecium $\mathrm{CC} 17$ and CC94 were different. Two main divergence events have occurred in the evolutionary path of E. faecium and the creation of E. faecium CC17. The first divergence event was estimated to have occurred approximately $2,776 \pm 818$ years ago, concomitant with the increased urbanization and domestication of animals. During this bifurcation, E. faecium species were classified into human and animal dominant lineages. The animal lineage was further categorized into an epidemic hospital lineage (E. faecium CC17) and a lineage that colonized and spread in communities only, causing sporadic infections in animals and humans (E. faecium CC94). The second divergence event was thought to have occurred because of the invention and use of antimicrobials in medicine and agriculture approximately $74 \pm 30$ years ago (5). These findings illustrated that the formation of $E$. faecium CC17 occurred in parallel with human behavior changes.

Another model using a synonymous single 
nucleotide polymorphism (sSNP) molecular clock and estimations of Escherichia coli and Bacillus anthracis parameters predicted that the evolutionary division of E. faecium CC17 and CC94 occurred 1,000,000 to $3,000,000$ years ago. Core genome analysis showed that the differences between the two subpopulations occurred at the core genomic level and long preceded the modern antibiotic era, or even long preceded the inhabitation of humans on earth (G). Admixture analysis showed a scarce number of recombination events between E. faecium CC17 and CC94.

A recent analysis of a global representative vancomycin-resistant Enterococcus (VRE) genome set estimated that the overall phylogenomic structure of vancomycin-resistant $E$. faecium $\mathrm{CC} 17$ was highly dependent on recombination (54\% of the genome). The split between E. faecium CC17 and CC94 was estimated to have occurred more than 2,765 years ago. Molecular clock calculations suggested that the branching of animal isolates and clinical lineages occurred approximately 502 years ago (7). However, the discrepancy is difficult to resolve using existing methods, since accurate phylogenomic analyses rely on the assumptions that recombination occurs across a restricted region of the genome and that these regions can be reliably detected and removed. E. faecium $\mathrm{CC} 17$ genomic analysis revealed recombination across the whole genome, even in relatively small sample subsets. Consequently, molecular clock approaches are prone to inaccuracies, thereby providing an explanation for wide discrepancies in estimates (8).

\section{SURVIVAL AND ADAPTATION IN HOSPITAL ENVIRONMENTS}

E. faecium CC17 is a typical example of a cumulative evolutionary process that improved the relative fitness of bacteria in hospital environments. The successful survival and spread of this lineage in hospital environments favors rapid adaptation to more antimicrobials, especially first-line clinical antimicrobials. The high genome plasticity of $E$. faecium $\mathrm{CC} 17$ is one of the key characteristics that may explain why it successfully adapted to harsh conditions, such as hospital environments, and how it managed antibiotic and antiseptic stresses. Recombination was found to have a significant impact on the E. faecium CC17 genome and the acquisition of antimicrobial resistance genes. Interestingly, E. faecium CC94 established an important reservoir for donating foreign
DNA to E. faecium CC17, and multiple recombinant regions comprise up to $26 \%$ of the E. faecium $\mathrm{CC} 17$ genome (9). The lack of clustered regularly interspaced short palindromic repeats-associated (CRISPR-cas) loci has also contributed to the adaptation of E. faecium CC17 in hospital environments. High recombination rates were commonly detected in vancomycin-resistant variants of hospital-adapted CC17 (10). Furthermore, MGEs play a crucial role in the environmental and nosocomial epidemic adaptation of hospital lineages. For example, vancomycin-resistant determinant van operons are always carried on the transposable element $\operatorname{Tn} 1549$, which accounts for the appearance and spread of E. faecium CC17 in hospital settings (11).

Phylogenetic and eBURST analyses of hospitaladapted E. faecium CC17 confirmed the existence of 3 separate hospital sub-lineages, originating from sequence types (STs) 17, 18, and 78 (12). The isolates originating from ST17 and ST18 contained a relatively high proportion of genomic text of pig isolates, while the ST78 lineage co-clustered with poultry-originating isolates (12).

E. faecium CC17 has several important clinical features, such as ampicillin resistance, vancomycin resistance, and the presence of the esp virulence factor, with the latter accounting for biofilm formation, urinary tract infections, and endocarditis. Once the $E$. faecium $\mathrm{CC} 17$ isolates acquired high invasive potential through horizontal gene transfer and adapted to a distinct pathogenic niche, the population was isolated and declined recombination with other populations (12). This corresponded with surveillant results, which indicated that hospital isolates commonly carried some resistance and virulence genes that were not detected in community/animal isolates (E. faecium CC94) (9).

\section{AMR OF E. FAECIUM CC17}

The worldwide ratio of $E$. faecalis-to-E. faecium infections in clinical settings has changed dramatically in favor of E. faecium CC17 after acquiring high resistance to multiple antimicrobials. In addition, the latter species is naturally resistant to cephalosporins and aminoglycosides at low levels, and the $\mathrm{CC} 17$ lineage is nearly always resistant to ampicillin (13). More importantly, vancomycin-resistant E. faecium CC17 has spread globally in the past few decades (14). As such, a better understanding of the resistance mechanisms of this pathogen is needed for the prediction and prevention of its dissemination. 
Resistance to ampicillin is a primary trait of $E$. faecium CC17. In the United States, nosocomial infections caused by ampicillin-resistant E. faecium CC17 increased in the 1980s, followed by the emergence of vancomycin-resistant $E$. faecium $\mathrm{CC} 17$ in the 1990s. In Europe, vancomycin-resistant E. faecium CC17 prevalence rates have been increasing since the 2000s. These findings strongly suggest that the emergence and spread of ampicillin-resistant $E$. faecium CC17 in hospitals has preceded the dramatic emergence of vancomycin-resistant E. faecium CC17. Hence, efforts for preventing the further spread of this epidemical pathogen should focus on the early disclosure of ampicillin-resistant E. faecium CC17 strains.

Ampicillin resistance in E. faecium CC17 is due to 1) alterations caused by mutations in penicillinbinding protein (PBP5), resulting in lower affinity; and 2) overproduction of PBP5 (5). E. faecium isolates of hospitals acquired selective advantage after obtaining ampicillin resistance and some virulence genes. After successfully exploiting the hospital environment, the adaptive isolates increased in frequency to become the dominant clones. By the "genetic capitalism" strategy, the dominant isolates acquired additional adaptive mechanisms more easily, such as vancomycin resistance, thereby fully adapting as a nosocomial pathogen that spread globally (15). Several studies have reported that the nucleotide difference of the PBP5 gene between ampicillin-resistant and -sensitive $E$. faecium isolates was 5\%. The mutation of the PBP5 gene may be the reason for the ampicillin resistance phenotype (6).

The van genes, especially van $A$, vanB, and van $M$, carry greater clinical significance, as they can confer intermediate-to-high levels of resistance to vancomycin and are encoded on MGEs. In some European countries, $30 \%$ to $50 \%$ of E. faecium $\mathrm{CC} 17$ isolates showed vancomycin resistance, and this was considered the greatest threat to successful clinical treatment. In China, the prevalence of vancomycin-resistant $E$. faecium (VREfm) has been considered as low as 3.6\% according to the report from 2010 China Antimicrobial Surveillance Network (CHINET). However, a monitoring data covering 45 tertiary hospitals indicated that the incidence of VREfm had increased to $14.3 \%$ in 2013 (16). Due to the conjugative transposons and plasmids, the dissemination of vancomycin resistance was expanded among enterococcal strains, species, and even genera such as Staphylococcus aureus. Consequently, VREfm are already the second most common nosocomial pathogen in the United States after heavy use of vancomycin in clinical settings (4).

Similarly in Europe, VREfm colonization and infection dramatically increased over a short period of time. However, unlike in the United States, VREfm colonization was limited in hospitals, and large community spreading was thought to be one reason for the sudden increase in VREfm colonization and infection. In the late 1980s, farmers in Europe began adding to animal feed avoparcin, a glycopeptide antimicrobial-like vancomycin. After this, VREfm colonization was soon observed in farm animals as well as in the community. The use of avoparcin in the animal industry was subsequently banned in Europe in 1996. However, persistent VREfm colonization in poultry has been reported up to eight years after the ban (17).

The optimal therapy for VRE infections in clinical settings remains uncertain. The new antibiotics daptomycin and linezolid are the most utilized last-line antibiotics. However, mutations in any one of the three genes, liaF, liaS, and liaR, have been linked to daptomycin resistance, while mutations in $23 \mathrm{~S}$ rRNA, the Cfr rRNA methyltransferase gene, or optrA have been reported to cause linezolid resistance. Although plasmid-mediated linezolid resistance can lead to sporadic outbreaks, resistance to last-line antibiotics remains uncommon (8).

Transferable resistance poses a great threat, as it can produce a much greater threat due to its wide and rapid dissemination. Several reports have suggested that the acquisition of insertion sequence (IS) elements can facilitate the niche adaptation of E. faecium CC17 by increasing its genome plasticity. These findings indicate that the global emergence of E. faecium $\mathrm{CC} 17$, as observed since 1990 , represents the evolution of the CC17 lineage with better adaptation (AMR) than other E. faecium lineages to the constraints of hospital environments (10).

\section{COLONIZATION AND VIRULENCE OF E. FAECIUM CC17}

E. faecium CC17 can infect or persistently colonize human hosts depending on progressively acquired genetic elements that confer selective advantages. These acquired genetic elements include the antimicrobial resistant genes (ARGs) and virulence genes. In addition to the ARGs, the colonization and virulence genes are 
also important for the adaptability and spread of $E$. faecium CC17 to hospital environments and/or patient niches. These colonization and/or virulence genes mainly include the fms genes encoding microbial surface components that recognize adhesive matrix molecules, esp $p_{\mathrm{Efm}}$ genes encoding surface proteins responsible for biofilm formation, and $h y l_{\text {Efm }}$ genes encoding putative glycoside hydrolases that facilitate intestinal colonization and peritoneal invasion (18). These virulence genes are often co-localized in putative pathogenicity islands (PAIs) or mobile elements, thereby facilitating their spread between isolates. PAIs are large elements that can be acquired by horizontal transfer and confer virulence to bacterial pathogens. The VREfm of 45 tertiary hospitals monitored in 2013 contained the $e^{2} p_{\mathrm{Efm}}$ gene with the frequencies of $89.9 \%$ (62/69), while $27.5 \%(19 / 69)$ of the VREfm strains carried $h y l_{\mathrm{Efm}}$ gene (16). The third-generation cephalosporins resistant $E$. faecium $\mathrm{CC} 17$ increased the risk of colonization and infection in hospitals. In hospitals, E. faecium was found to remain viable on inanimate surfaces from 7 days to 2 months, which increases the risk of acquiring ARGs and virulence genes (1).

\section{SUMMARY}

Multiple AMR E. faecium CC17 outbreaks have not only incurred significant costs for healthcare systems but also placed vulnerable patients at higher risk of acquiring fatal infections. The patterns of E. faecium CC17 variation illustrate that new phenotypes are likely to continue to emerge, driven by local variations in selective stress and access to distinct gene pools via both homologous recombination and an extensively mobilizable pangenome. These genomic features suggest that controlling the hospital spread of $E$. faecium CC17 will remain challenging. The successful control of AMR E. faecium CC17 outbreaks often mentions the importance of general infection control procedures, such as education for healthcare workers, sanitation of hands and environments, antimicrobial stewardship, and use of sterile equipment and personal protective gear. The use of molecular typing, rapid van gene detection, and AMR surveillance can help to identify outbreaks early, allowing infection control to limit the spread of the outbreak. Antimicrobial stewardship practices can limit the dissemination of antimicrobial resistance genes in E. faecium CC17, extending the efficacy of current antimicrobials.
Conflicts of interest: No conflicts of interest. Funding: Supported by Chinese Academy of Medical Science (CAMS) Innovation Fund for Medical Science (CIFMS 2019-12M-5-024) and National Natural Science Foundation of China (32172314).

doi: $10.46234 / \mathrm{ccdcw} 2021.277$

\# Corresponding author: Dajin Yang, yangdajin@cfsa.net.cn.

${ }^{1}$ NHC Key Laboratory of Food Safety Risk Assessment, Chinese Academy of Medical Science Research Unit (2019RU014), China National Center for Food Safety Risk Assessment, Beijing, China.

Submitted: December 01, 2021; Accepted: December 21, 2021

\section{REFERENCES}

1. Lee T, Pang S, Abraham S, Coombs GW. Antimicrobial-resistant CC17 Enterococcus faecium: the past, the present and the future. J Glob Antimicrob Resist 2019;16:36-47. http://dx.doi.org/10.1016/j.jgar. 2018.08.016.

2. Caballero S, Kim S, Carter RA, Leiner IM, Sušac B, Miller L, et al. Cooperating commensals restore colonization resistance to vancomycinresistant Enterococcus faecium. Cell Host Microbe 2017;21(5):592 602.e4. http://dx.doi.org/10.1016/j.chom.2017.04.002.

3. Palmer KL, Godfrey P, Griggs A, Kos VN, Zucker J, Desjardins C, et al. Comparative genomics of enterococci: variation in Enterococcus faecalis, clade structure in $E$. faecium, and defining characteristics of $E$. gallinarum and E. casseliflavus. mBio 2012;3(1):e00318 - 11. http://dx. doi.org/10.1128/mBio.00318-11.

4. Huang JH, Wang ML, Gao Y, Chen L, Wang LP. Emergence of plasmid-mediated oxazolidinone resistance gene poxtA from CC17 Enterococcus faecium of pig origin. J Antimicrob Chemother 2019;74(9):2524 - 30. http://dx.doi.org/10.1093/jac/dkz250.

5. Lebreton F, van Schaik W, McGuire AM, Godfrey P, Griggs A, Mazumdar V, et al. Emergence of epidemic multidrug-resistant Enterococcus faecium from animal and commensal strains. $\mathrm{mBio}$ 2013;4(4):e00534 - 13. http://dx.doi.org/10.1128/mBio.00534-13.

6. Galloway-Pena J, Roh JH, Latorre M, Qin X, Murray BE. Genomic and SNP analyses demonstrate a distant separation of the hospital and community-associated clades of Enterococcus faecium. PLoS One 2012;7(1):e30187. http://dx.doi.org/10.1371/journal.pone.0030187.

7. Rios R, Reyes J, Carvajal LP, Rincon S, Panesso D, Echeverri AM, et al. Genomic epidemiology of vancomycin-resistant Enterococcus faecium (VREfm) in Latin America: revisiting the global VRE population structure. Sci Rep 2020;10(1):5636. http://dx.doi.org/10.1038/s41598020-62371-7.

8. van Hal SJ, Willems RJL, Gouliouris T, Ballard SA, Coque TM, Hammerum AM, et al. The global dissemination of hospital clones of Enterococcus faecium. Genome Med 2021;13(1):52. http://dx.doi.org/ 10.1186/s13073-021-00868-0.

9. de Been M, van Schaik W, Cheng L, Corander J, Willems RJ. Recent recombination events in the core genome are associated with adaptive evolution in Enterococcus faecium. Genome Biol Evol 2013;5(8):1524 35. http://dx.doi.org/10.1093/gbe/evt111.

10. Pinholt M, Gumpert H, Bayliss S, Nielsen JB, Vorobieva V, Pedersen M, et al. Genomic analysis of 495 vancomycin-resistant Enterococcus faecium reveals broad dissemination of a vanA plasmid in more than 19 clones from Copenhagen, Denmark. J Antimicrob Chemother 2017;72(1):40 - 7. http://dx.doi.org/10.1093/jac/dkw360.

11. van Hal SJ, Ip CLC, Ansari MA, Wilson DJ, Espedido BA, Jensen SO, et al. Evolutionary dynamics of Enterococcus faecium reveals complex genomic relationships between isolates with independent emergence of vancomycin resistance. Microb Genom 2016;2(1):e000048. http://dx. 
doi.org/10.1099/mgen.0.000048.

12. Willems RJL, Top J, van Schaik W, Leavis H, Bonten M, Sirén J, et al. Restricted gene flow among hospital subpopulations of Enterococcus faecium. mBio 2012;3(4):e00151 - 12. http://dx.doi.org/10.1128/ mBio.00151-12.Print2012.

13. Cattoir V, Giard JC. Antibiotic resistance in Enterococcus faecium clinical isolates. Expert Rev Anti Infect Ther 2014;12(2):239 - 48. http: //dx.doi.org/10.1586/14787210.2014.870886.

14. O'Driscoll T, Crank CW. Vancomycin-resistant enterococcal infections: epidemiology, clinical manifestations, and optimal management. Infect Drug Resist 2015;8:217 - 30. http://dx.doi.org/10. 2147/IDR.S54125.

15. Raven KE, Gouliouris T, Brodrick H, Coll F, Brown NM, Reynolds R, et al. Complex routes of nosocomial vancomycin-resistant Enterococcus faecium transmission revealed by genome sequencing. Clin Infect Dis
2017;64(7):886 - 93. http://dx.doi.org/10.1093/cid/ciw872.

16. Yang JX, Li T, Ning YZ, Shao DH, Liu J, Wang SQ, et al. Molecular characterization of resistance, virulence and clonality in vancomycinresistant Enterococcus faecium and Enterococcus faecalis: a hospital-based study in Beijing, China. Infect Genet Evol 2015;33:253 - 60. http://dx. doi.org/10.1016/j.meegid.2015.05.012.

17. Zhou XW, Willems RJL, Friedrich AW, Rossen JWA, Bathoorn E. Enterococcus faecium: from microbiological insights to practical recommendations for infection control and diagnostics. Antimicrob Resist Infect Control 2020;9(1):130. http://dx.doi.org/10.1186/ s13756-020-00770-1.

18. Gao W, Howden BP, Stinear TP. Evolution of virulence in Enterococcus faecium, a hospital-adapted opportunistic pathogen. Curr Opin Microbiol 2018;41:76 - 82. http://dx.doi.org/10.1016/j.mib.2017.11. 030. 